\title{
THE SUM-PRODUCT SPLTTING PROPERTY AND INJECTIVE DIRECT SUMS OF MODULES OVER VON NEUMANN REGULAR RINGS
}

\author{
BIRGE ZIMMERMANN-HUISGEN
}

\begin{abstract}
ABSTRACr. Let $\left(M_{i}\right)_{i \in I}$ be a family of modules over a von Neumann regular ring. It is shown that for the splitness of the canonical inclusion $\bigoplus_{i \in I} M_{i} \subset \mathbb{I}_{i \in I} M_{i}$ it is necessary and sufficient that there be a finite subset $I^{\prime}$ of $I$ such that the restricted sum $\bigoplus_{i \in I I^{\prime}} M_{i}$ is semisimple with finitely many homogeneous components, all simple summands being finite dimensional over their endomorphism rings. This yields a characterization of those families of injectives whose direct sum is again injective.
\end{abstract}

Which families $\left(M_{i}\right)_{i \in I}$ of modules over a von Neumann regular ring $R$ have the property that the direct sum $\bigoplus_{i \in I} M_{i}$ is injective? Levine [6, Theorem 2.6] has shown that, if the $M_{i}$ 's are injective hulls of simple modules $S_{i}$ respectively, then $\bigoplus_{i \in I} M_{i}$ is injective if and only if the family $\left(S_{i}\right)_{i \in I}$ contains only a finite number of isomorphism types and each module $S_{i}$ which occurs an infinite number of times is finite dimensional over its endomorphism ring. The following adjacent result has been obtained by Faith [1, p. 159]: For an $R$-module $M$ to be $\Sigma$-injective (i.e. all direct sums of copies of $M$ are injective) it is necessary and sufficient that $M$ be semisimple and finitely generated over its endomorphism ring.

The theorem which follows answers completely the question raised above. In addition, it concludes previous investigations on the splitting of the canonical injection $\bigoplus_{i \in I} M_{i} \hookrightarrow \Pi_{i \in I} M_{i}$ (see [3]-[5], [8]) in the case of a von Neumann regular ring. The problem of whether splitness of this sequence forces almost all $M_{i}$ 's to be direct sums of simple modules had been left open. The answer is positive.

If not indicated otherwise, $R$ denotes an associative (not necessarily commutative) von Neumann regular ring with identity, and " $R$-module" means unitary right $R$-module.

THEOREM. For each family $\left(M_{i}\right)_{i \in I}$ of $R$-modules the following statements are equivalent:

(1) $\bigoplus_{i \in I} M_{i}$ is a direct summand of $\prod_{i \in I} M_{i}$ in the canonical embedding.

(2) There is a finite subset $I^{\prime}$ of $I$ such that $\bigoplus_{i \in I \backslash I^{\prime}} M_{i}$ is injective.

(3) There is a finite subset $I^{\prime}$ of $I$ such that $\bigoplus_{i \in I I^{\prime}} M_{i}$ is a direct sum of simple modules, each of which is finite dimensional over its endomorphism ring, and such that the number of homogeneous components of $\bigoplus_{i \in I I^{\prime}} M_{i}$ is finite.

Received by the editors October 18, 1979.

AMS (MOS) subject classifications (1970). Primary 16A30, 16A52.

(C) 1981 American Mathematical Society 0002-9939/81/0000-0456/\$02.00 
COROllary 1. Given a family $\left(M_{i}\right)_{i \in I}$ of $R$-modules, the direct sum $\bigoplus_{i \in I} M_{i}$ is injective precisely if the following two conditions are satisfied:

(a) Each $M_{i}$ is injective.

(b) There is a finite subset $I^{\prime}$ of $I$ and a finite number of simple $R$-modules $U_{1}, \ldots, U_{n}$, each of finite dimension over its endomorphism ring, such that $\bigoplus_{i \in I I^{\prime}} M_{i}$ is a direct sum of copies of the $U_{j}^{\prime}$ s.

It is well known that the rings for which the class of injective modules is closed under direct sums are precisely the right noetherian ones. The theorem enables us to describe the other extreme. The prototype of a regular ring without (nontrivially) infinite, injective direct sums is the endomorphism ring of an infinite dimensional vector space:

COROllary 2. For the existence of an infinite family of nonzero right $R$-modules whose direct sum is injective it is necessary and sufficient that there is a right primitive ideal $\mathrm{r}$ so that $R / \mathrm{r}$ is artinian. (Note that the latter condition is left-right symmetric.)

We now prepare for the proof of " $(1) \Rightarrow(3)$ " of the theorem, the other implications being well known as indicated above. There is an amusing twist in the argument which we will give: Assuming (1) and the contrary of (3) we will construct a family of orthogonal idempotents of $R$ violating the conclusion of the lemma below, by using an infinite number of times the fact that such a family cannot exist.

LEMMA. ${ }^{1}$ Let $R$ be an arbitrary unital ring (for a short moment), $\left(M_{i}\right)_{i \in I}$ a family of $R$-modules and $E$ a set of orthogonal idempotents of $R$. Moreover suppose that $\bigoplus_{i \in I} M_{i}$ is a direct summand of $\Pi_{i \in I} M_{i}$ with respect to the canonical embedding. Then there are finite subsets $I^{\prime}$ of $I$ and $E^{\prime}$ of $E$ with $\left(\bigoplus_{i \in I \backslash I^{\prime}} M_{i}\right) e=0$ for $e \in E \backslash E^{\prime}$.

Proof. Assume the contrary. Then there are sequences $\left(i_{n}\right)_{n \in N}$ resp. $\left(e_{n}\right)_{n \in N}$ of distinct elements of $I$ resp. $E$ with $M_{i_{n}} e_{n} \neq 0$ for $n \in N$. Since the hypothesis implies that $\bigoplus_{n \in N} M_{i_{n}}$ is a direct summand of $\Pi_{n \in N} M_{i_{n}}$, we may identify $i_{n}$ with $n$ and $I$ with $\mathrm{N}$ for simplicity. Let $q$ be a projection of $\Pi_{n \in \mathrm{N}} M_{n}$ onto $\bigoplus_{n \in \mathrm{N}} M_{n}$ along a direct complement, $C$ say, and by $q_{i}$ denote the canonical projection $\bigoplus_{n \in \mathrm{N}} M_{n} \rightarrow M_{i}$. Moreover, given an $R$-module $X$, write $A_{n}(X)$ for the annihilator of $\left\{e_{1}, \ldots, e_{n}\right\}$ in $X$.

By construction we have $A_{n-1}\left(M_{n}\right) \not \subset A_{n}\left(M_{n}\right)$ for each $n$. Pick

$$
x_{n} \in A_{n-1}\left(M_{n}\right) \backslash A_{n}\left(M_{n}\right)
$$

and set $x=\left(x_{n}\right)_{n \in \mathrm{N}} \in \Pi_{n \in \mathrm{N}} M_{n}$. We now follow the line of [8, proof of Lemma 3.2]: Writing $s_{n}=\left(x_{1}, \ldots, x_{n}, 0, \ldots\right)$ and $x-s_{n}=t_{n}+c_{n}$ with $t_{n} \in \bigoplus_{n \in N} M_{n}$ and $c_{n} \in C$, we obtain $q(x)=s_{n}+t_{n}$. Now choose a natural number $m$ such that

\footnotetext{
${ }^{1}$ The referee has kindly pointed out to us that the lemma is an immediate consequence of a result of Sarath and Varadarajan [7, Proposition 1].
} 
$q_{m} q(x)=0$. From $x-s_{m} \in \Pi_{n \in \mathrm{N}} A_{m}\left(M_{n}\right)=A_{m}\left(\Pi_{n \in \mathrm{N}} M_{n}\right)$ we infer that

$$
t_{m}=(1-q)\left(x-s_{m}\right) \in A_{m}(C) \subset A_{m}\left(\prod_{n \in N} M_{n}\right)
$$

and conclude that $x_{m}=q_{m} s_{m}=-q_{m} t_{m} \in A_{m}\left(M_{m}\right)$, which contradicts the choice of $x_{m}$.

PROOF OF THE THEOREM. (1) $\Rightarrow(3)$. Since otherwise there is nothing to prove, we assume that $I$ is infinite. The principal step will be to show that $R / \operatorname{Ann}_{R} M_{j}$ is artinian for some $j \in I$. We will proceed by assuming the contrary and inductively constructing sequences $\left(i_{n}\right)_{n \in N}$ resp. $\left(e_{n}\right)_{n \in N}$ of distinct elements of $I$ resp. pairwise orthogonal idempotents of $R$ with $M_{i_{n}} e_{n} \neq 0$. On the other hand, the lemma tells us that such sequences do not exist.

Start with an arbitrary element $i_{1}$ of $I$. Since, by assumption, $R / \operatorname{Ann}_{R} M_{i_{1}}$ is not artinian, there exists an infinite set $E_{1}$ of orthogonal idempotents of $R$, none of which annihilates $M_{i_{1}}$ (see [2, Proposition 2.18]). Now the lemma yields a finite subset $I_{1}$ of $I$ and a finite subset $F_{1}$ of $E_{1}$ such that $\left(\bigoplus_{i \in I I_{1}} M_{i}\right)\left(E_{1} \backslash F_{1}\right)=0$. Pick $e_{1} \in E_{1} \backslash F_{1}$. Next, suppose that $i_{1}, \ldots, i_{n}$ and $e_{1}, \ldots, e_{n}$ are as desired and that $I_{1} \subset I_{2} \subset \cdots \subset I_{n}$ are finite subsets of $I$ with $\left(\bigoplus_{i \in I I_{k}} M_{i}\right) e_{k}=0$ for $1<k<n$ (which implies $i_{k} \in I_{k}$ ). Define $f=1-e_{1}-\cdots-e_{n}$ and note that $\bigoplus_{i \in I I_{n}} M_{i}$ and $\Pi_{i \in I \backslash I_{n}} M_{i}$ are $f R f$-modules. In particular, for $i \in I \backslash I_{n}$ the rings $R / \operatorname{Ann}_{R} M_{i}$ and $f R f / \mathrm{Ann}_{f R f} M_{i}$ are isomorphic. Since $I$ is infinite, we can choose $i_{n+1} \in I \backslash I_{n}$. Using the fact that $\bigoplus_{i \in I I_{n}} M_{i}$ is a direct summand of $\Pi_{i \in I V_{n}} M_{i}$ and repeating the argument above, we obtain an infinite set $E_{n+1}$ of orthogonal idempotents of $f R f$ with $M_{i_{n+1}} e \neq 0$ for $e \in E_{n+1}$, together with finite subsets $I_{n+1}$ of $I$ resp. $F_{n+1}$ of $E_{n+1}$ such that $\left(\bigoplus_{i \in I \backslash I_{n+1}} M_{i}\right)\left(E_{n+1} \backslash F_{n+1}\right)=0$. Naturally, we are free to assume $I_{n} \subset I_{n+1}$. If we pick $e_{n+1} \in E_{n+1} \backslash F_{n+1}$, the induction hypothesis is clearly established for $n+1$.

Since $\bigoplus_{i \in J} M_{i}$ is a direct summand of $\Pi_{i \in J} M_{i}$ for each subset $J$ of $I$, we have even shown that almost all-for the remainder of the proof we may assume all for simplicity-of the rings $R / \mathrm{Ann}_{R} M_{i}$ are artinian and hence semisimple. Consequently, $\bigoplus_{i \in I} M_{i}$ is a direct sum of simple $R$-modules which are finite dimensional over their endomorphism rings, and each $M_{i}$ has only a finite number of homogeneous components. If the number of homogeneous components of $\bigoplus_{i \in I} M_{i}$ were infinite, we could choose a sequence $\left(i_{n}\right)_{n \in \mathrm{N}}$ of elements of $I$ such that each $M_{i_{n+1}}$ contains a simple module whose isomorphism type does not appear in $\bigoplus_{k<n} M_{i_{k}}$. Partition $\left\{i_{n}: n \in \mathbf{N}\right\}$ into an infinite family $\left(J_{n}\right)_{n \in \mathbf{N}}$ of pairwise disjoint infinite subsets and define $\bar{M}_{n}=\bigoplus_{i \in J_{n}} M_{i}$. Clearly, $\bigoplus_{n \in \mathrm{N}} \bar{M}_{n}$ is again a direct summand of $\Pi_{n \in \mathrm{N}} \bar{M}_{n}$, whereas none of the rings $R / \operatorname{Ann}_{\bar{M}_{n}} R$ is artinian. But this is impossible by the preceding paragraph.

$(3) \Rightarrow(2)$ is known (see [1, p. 159]), but we will include a short proof for completeness. Write $\bigoplus_{i \in I I^{\prime}} M_{i} \cong \bigoplus_{\text {fin }} S_{k}^{\left(L_{k}\right)}$, where $I^{\prime}$ is a finite subset of $I$ and each $S_{k}$ is a simple module which is finite dimensional over its endomorphism ring. Since $R_{k}=R / \operatorname{Ann}_{R} S_{k}$ is semisimple, $S_{k}^{\left(L_{k}\right)}$ is an injective $R_{k}$-module, and hence even an injective $R$-module because $R_{k}$ is $R$-flat. Consequently, $\bigoplus_{i \in I I^{\prime}} M_{i}$ is injective.

$(2) \Rightarrow(1)$ is clear. 


\section{REFERENCES}

1. C. Faith, Modules finite over endomorphism ring, Lecture Notes in Math., vol. 246, Springer-Verlag, Berlin and New York, 1972.

2. K. R. Goodearl, Von Neumann regular rings, Pitman, New York, 1979.

3. P. A. Griffith, A note on a theorem of Hill, Pacific J. Math. 29 (1969), 279-284.

4. L. Gruson and C. U. Jensen, Deux applications de la notion de L-dimension, C. R. Acad. Sci. Paris Sér. A 282 (1976), 23-24.

5. H. Lenzing, Direct sums of projective modules as direct summands of their direct product, Comm. Algebra 4 (1976), 681-691.

6. J. Levine, On the injective hulls of semisimple modules, Trans. Amer. Math. Soc. 135 (1971), 115-126.

7. B. Sarath and K. Varadarajan, Injectivity of direct sums, Comm. Algebra 1 (1974), 517-530.

8. W. Zimmermann, Rein injektive direkte Summen von Moduln, Comm. Algebra 5 (1977), 1083-1117.

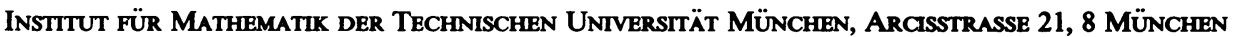
2, Federal Republic of Germany 